\title{
Conversion of a polarization microscope into a Mueller matrix microscope. Application to the measurement of textile fibers
}

\section{Conversión de un microscopio de polarización en un microscopio de matriz de Mueller. Aplicación a la medida de fibras textiles.}

\author{
E. Kuntman, ${ }^{1}$ O. Arteaga, ${ }^{1,}{ }^{*}$ J. Antó, ${ }^{1}$ D. Cayuela, ${ }^{2}$ E. Bertran ${ }^{1}$ \\ 1. Dep. de Física Aplicada i Òptica, C/Martí i Franquès, Universitat de Barcelona, \\ 08030, Barcelona, Spain \\ 2. Intexter, Universitat Politècnica de Catalunya, C/ Colom 15, 08222, Terrassa, Spain \\ (*) E-mail: oarteaga@ub.edu
}

Received: 18/06/2015 Accepted: 26/11/2015

DOI: $10.7149 /$ OPA.48.4.309

\begin{abstract}
:
This work reports the conversion of a commercial polarization microscope (Zeiss Jenaval) into an automatized Mueller matrix microscope. A Mueller matrix measurement provides the value of all the optical properties of a specimen (linear dichroism, linear birefringence, circular dichroism etc.) at a specific wavelength. In contrast to traditional polarization microscopes, which use white light and crossed polarizers to generate colored interference patterns that are analyzed by the microscope user and give only semi-quantitative results, here we demonstrate that it is possible to convert a polarization microscope into a Mueller microscope by only adding two motorized rotating compensators into the optical path without altering the optics or other opto-mechanical elements of the microscope. To our knowledge this is the most compact, fast and handy Mueller microscope ever developed. The performance of this new Mueller matrix microscope is illustrated with some birefringence measurements on textile fibers.
\end{abstract}

Key words: microscopy, polarimetry, Mueller matrix, birefringence.

\section{RESUMEN:}

Este trabajo describe la conversión de un microscopio de polarización comercial (Zeiss Jenaval) en un microscopio de matriz de Mueller automatizado. Una medida de la matriz de Mueller proporciona todas las propiedades ópticas de una muestra (dicroísmo lineal, birrefringencia lineal, dicroísmo circular, etc) para una longitud de onda dada. Los microscopios de polarización tradicionales usan luz blanca y polarizadores cruzados para generar un patrón de colores interferencia que debe ser interpretado por el usuario del microscopio y proporciona resultados sólo semi-cuantitativos. En este trabajo demostramos que añadiendo dos compensadores rotatorios actuados por sendos motores es posible convertir un microscopio de polarización en un microscopio de matriz de Mueller sin alterar la óptica u otros elementos opto-mecánicos del microscopio. Creemos que este es el microscopio de matriz de Mueller más compacto, rápido y práctico que se ha desarrollado. El funcionamiento de este microscopio se demuestra con algunas medidas de birrefringencia en fibras textiles.

Palabras clave: microscopía, polarimetría, matriz de Mueller, birrefringencia. 


\section{REFERENCES AND LINKS / REFERENCIAS Y ENLACES}

[1] E. Bernabeu, J. J. Gil, "An experimental device for the dynamic determination of Mueller matrices,” J. Opt. 16, 139-141 (1985).

http://dx.doi.org/10.1088/0150-536X/16/3/006

[2] K. Ichimoto, K. Shinoda, T. Yamamoto, J. Kiyohara, "Photopolarimetric measurement system of Mueller matrix with dual rotating waveplates," Publ. Nat. Ast. Obs. J. 9, 11-19 (2006).

[3] O. Arteaga, M. Baldrís, J. Antó, A. Canillas, E. Pascual, E. Bertran, "Mueller matrix microscope with a dual continuous rotating compensator setup and digital demodulation," Appl. Opt. 53, 2236-2245 (2014). http://dx.doi.org/10.1364/A0.53.002236

[4] O. Arteaga, E. Kuntman, "Beyond polarization microscopy: Mueller matrix microscopy with frequency demodulation," in Proceedings SPIE 9099, Polarization: Measurement, Analysis, and Remote Sensing XI, 90990R, (2014).

[5] O. Arteaga, J. Freudenthal, B. Wang, B. Kahr, "Mueller matrix polarimetry with four photoelastic modulators: theory and calibration," Appl. Opt. 51, 6805-6817 (2012). http://dx.doi.org/10.1364/A0.51.006805

[6] A. De Martino, Y.-K. Kim, E. Garcia-Caurel, B. Laude, B. Drévillon, “Optimized Mueller polarimeter with liquid crystals," Opt. Lett. 28, 616-618 (2003). http://dx.doi.org/10.1364/OL.28.000616

[7] R. M. A. Azzam, "Division-of-amplitude photopolarimeter (DOAP) for the simultaneous measurement of all four stokes parameters of light," Opt. Acta 29, 685-689 (1982). http://dx.doi.org/10.1080/713820903

[8] D. Lara, C. Dainty, “Axially resolved complete Mueller matrix confocal microscopy,” Appl. Opt. 45, 1917-1930 (2006). http://dx.doi.org/10.1364/A0.45.001917

[9] 0. Arteaga, and A. Canillas, "Analytic inversion of the Mueller-Jones polarization matrices for homogeneous media," Opt. Lett. 35, 559-561, (2010). http://dx.doi.org/10.1364/OL.35.000559

[10] L. C. Sawyer, Polymer Microscopy, Polymer Microscopy. Third Edition. L.C. Sawyer, D.T. Grubb, and G.F. Meyers. Springer, New York; 2008. ISBN 978-0-387-72627-4

[11] V. Devlaminck, R. Ossikovski, "Uniqueness of the differential Mueller matrix of uniform homogeneous media," Opt. Lett. 39, 3149-3152 (2014). http://dx.doi.org/10.1364/OL.39.003149

[12] O. Arteaga, A. Canillas, G. E. Jellison, "Determination of the components of the gyration tensor of quartz by oblique indicence transmission two-modulator generalized ellipsometry," Appl. Opt. 48, 5307-531, (2009).

http://dx.doi.org/10.1364/A0.48.005307

[13] J. J. Gil, E. Bernabeu, “A depolarization Criterion in Mueller matrices,” Opt. Acta 32, 259-262, (1985).

http://dx.doi.org/10.1364/A0.48.005307

\section{Introduction}

For decades microscopy techniques for measuring optical anisotropy have hardly been altered. Conventional techniques use white light and crossed polarizers which lead to colored interference patterns in the sample image. Recently, we combined methods and techniques developed in the field of polarimetry $[1,2]$ with space resolved measurements to develop a new prototype Mueller matrix microscope [3]. This new instrument uses monochromatic light together with a dual rotating compensator setup to carry out a quantitative analysis of optical anisotropy of the sample in transmission or in reflection (epi-illumination) $[3,4]$. The dual rotating compensator setup is one of the most popular approaches to measure the complete Mueller matrix, but there several other alternatives discussed in Refs. [5-8]. 
The Mueller matrix (MM) microscope produces 16 images of a sample in around one minute. Each one of these images corresponds to a different element of the $4 \times 4$ Mueller matrix, providing the complete quantitative analysis of changes in the polarization state of the light as being transmitted or reflected. The MM microscope is able to measure the magnitude and orientation of linear dichroism and linear birefringence, the circular birefringence, the circular dichroism and to evaluate also the depolarization.

This paper reports an upgraded version of our previously described Mueller matrix microscope [3]. Our new setup is based on a commercial polarization microscope that has been modified to do Mueller matrix measurements. We illustrate the performance of the Mueller microscope with birefringence measurements in textile fibers.

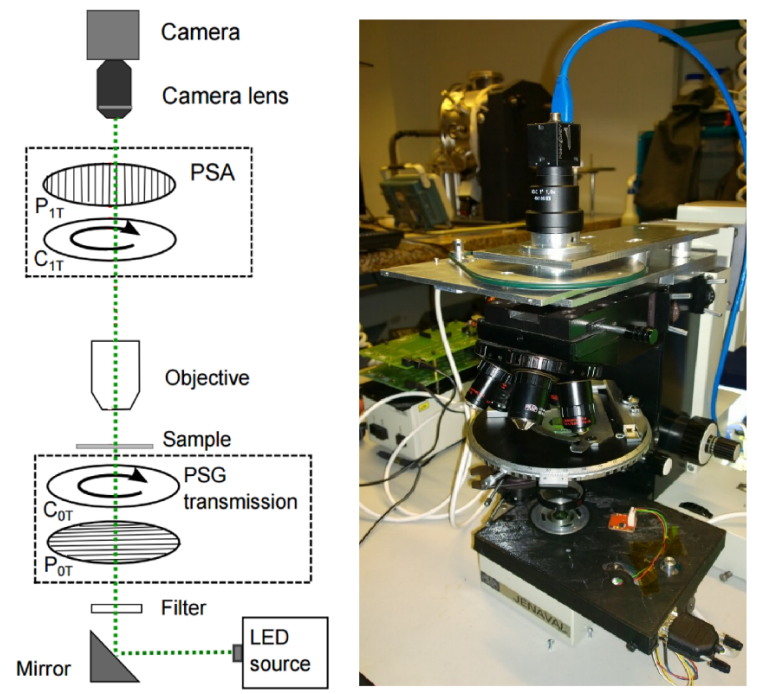

Fig. 1. Scheme of the setup for the microscope (left) and photo of our polarization microscope after conversion into a Mueller matrix microscope (right).

\section{Technical modifications}

Our approach is to determine the MM of the sample within a microscopic resolution. The images are continuously captured by a CMOS camera while two rotating compensators modulate the polarization of light $[9,10]$. The images are frequency analyzed at the pixel level by digital demodulation as the intensity is detected [3]. The determination of the optical properties keeps the same high lateral resolution of standard microscopy measurements. The key point necessary to modify a polarization microscope into a $\mathrm{MM}$ microscope is the addition of two continuously rotating compensators. One of them is placed before the sample and works as a polarization state generator (PSG), the other one is placed after the objective and works as a polarization state analyzer (PSA). The objectives that we use are for polarization microscopy hence, they are strain free and do not exhibit any significant amount of birefringence. The vertical spacing in a polarization microscope is very tight and the main challenge was to implement rotating compensators that were thin enough to fit into the microscope structure. We achieved this by using a belt-driven motor that rotates a thin metallic piece holding the polymer retarding film. The compensators are made of a $\lambda / 4$ polymer retarding film (Edmund Optics) with a thickness of only $75 \mu \mathrm{m}$ therefore, the effects of any misalignment with respect to the optical path is negligible. The retardance of the compensators for every wavelength of measurement (that is close to a $\lambda / 4$ but not perfect) is determined during de calibration process, as it was detailed in Ref. [3].

Fig.1 shows a photo of the modified Zeiss Jenaval polarization microscope. The original Halogen lamp has been replaced by a more stable LED source and band-pass filters are used to select the wavelength of light for each measurement. As seen in the photo, it is a compact tabletop instrument which can be operated by a technician without deep notions about the Mueller matrix polarimetry. 
The PSG and PSA motors use Arduino controllers with custom made software. The detector is a point Point Grey Flea3 CMOS camera. In our most usual configuration, it captures images at 50 fps. As the intensity is detected by the camera, the data pertaining to every individual pixel is frequency analyzed by digital demodulation. MM measurements maintain the same lateral resolution of standard polarization microscopy measurements. The compensator in the PSG rotates at approximately $0.0694 \mathrm{~Hz}$, while the compensator in the PSA rotates five times slower, at around $0.0138 \mathrm{~Hz}$. This ratio of angular speeds is not arbitrary, as we showed before [8], it provides the optimum conditions for the frequency demodulation. The whole instrument is controlled by a personal computer with custom-made LabView program. A complete imaging measurement of the space-resolved Mueller matrix takes around 1 minute.

\section{Measurements}

MM microscopy measurements provide information of all the optical properties of light as it propagates through a material, namely, the birefringence occurring when the beam propagates at different speeds depending on its polarization, and the dichroism when the absorption of the sample depends on the polarization state of the light. As the Mueller matrix provides a complete quantitative characterization of the sample, linear birefringence and linear dichroism effects can be well distinguished from the usually much weaker circular birefringence and circular dichroism effects. The algorithm that we use to recover all these optical effects from the Mueller matrix is based on an analytic inversion [9].

\subsection{Application to textile fibers}

Most textile fibers are linearly birefringent since the polymers comprising the fiber are highly oriented in the direction of the fiber axis [10]. Consequently, measurement of the birefringence of a fiber can provide information on the manner how fiber structure differs in directions parallel and perpendicular to the fiber axis and the values of birefringence measured in textile fibers are commonly used for fiber identification and quality control during fiber production.

MM microscopy in textile fibers provides an automatized and straightforward determination of its linear birefringence. With respect to a conventional determination of linear birefringence, it has the advantage that the fiber does not need to be oriented in any particular way. Fig. 2 shows a MM measure of a polyamide fiber at a wavelength of $620 \mathrm{~nm}$. For this horizontal orientation of the fiber, the presence of linear birefringence is clearly manifested in the non-vanishing values of MM elements $\mathrm{m}_{23}$ and $\mathrm{m}_{32}[11]$.

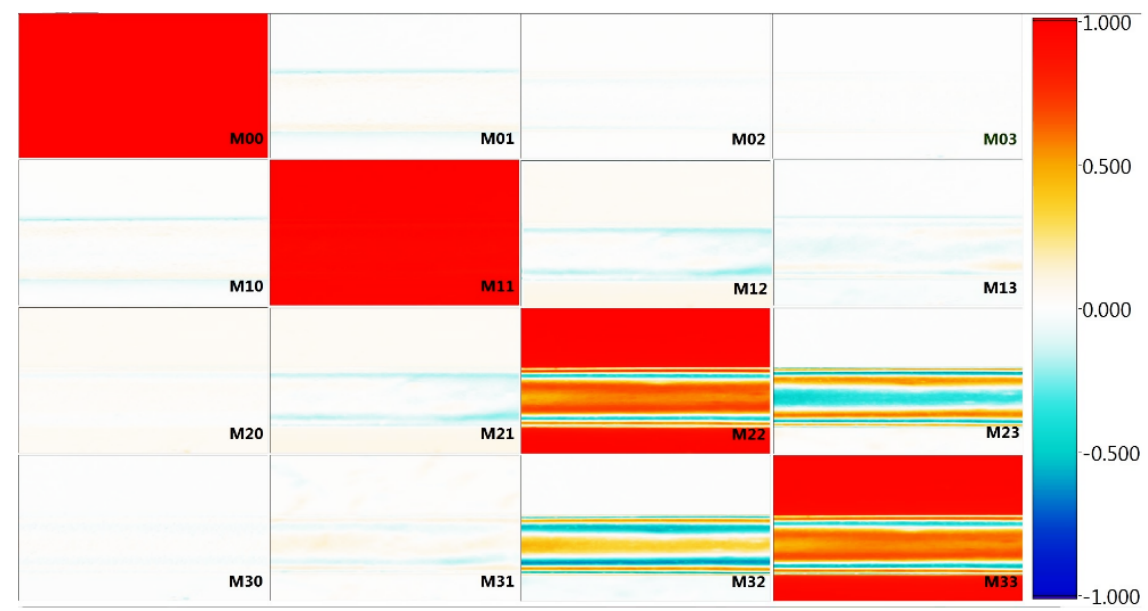

Fig 2. Normalized transmission Mueller matrix on a stretched polyamide textile fiber with $50 \mu \mathrm{m}$ diameter. The wavelength of measurement is $620 \mathrm{~nm}$. 


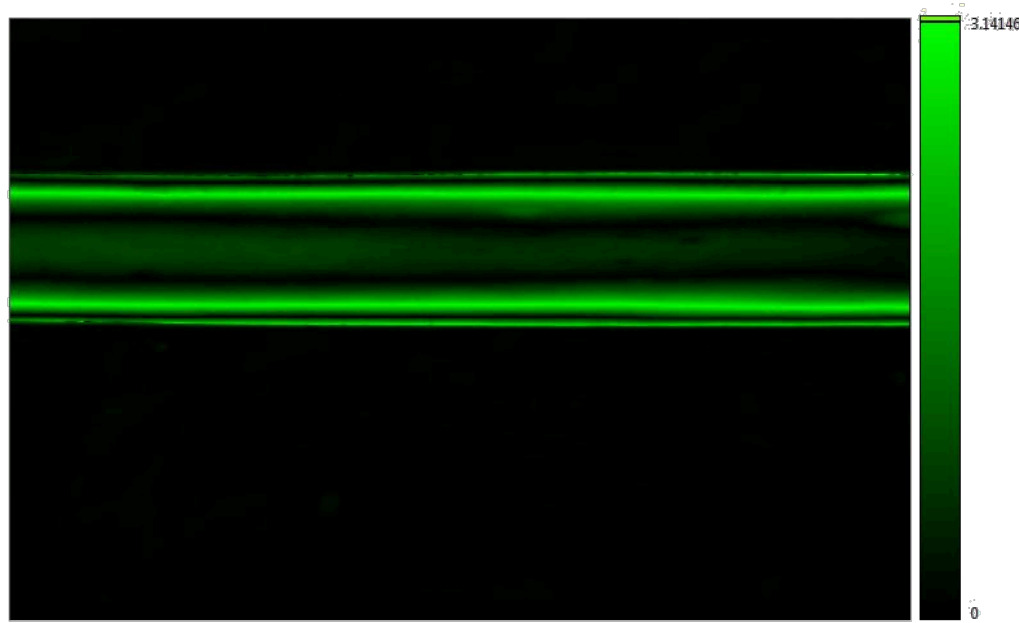

Fig 3. Magnitude of the linear birefringence in the fiber calculated from the Mueller matrix in Fig. 2. The lower refractive index corresponds to the direction of the fiber.

A map of the linear birefringence in this polyamide fiber is provided in Fig. 3, which is calculated from the Mueller Matrix [9]. However, for these types of fibers the linear birefringence tends to be quite large, sometimes crossing various orders of birefringence. This can be readily seen in Figs. 2 and 3 by the fringes or false color oscillations seen at the edges of the fiber. Therefore, the absolute magnitude of linear birefringence can only be recovered after a careful determination of the order of birefringence $[11,12]$.

The linear birefringence of a sample is given by the following formula:

$$
L B=\frac{2 \pi \Delta \mathrm{nd}}{\lambda}
$$

$\lambda$ is the wavelength of the light, $d$ is the thickness of the sample and $\Delta n$ is $n_{e}-n_{0}$, where $n_{e}$ and $n_{o}$ are the extraordinary and ordinary refractive indices respectively, which are wavelength dependent. For our concerns, at a given wavelength, LB is directly proportional to the thickness, d, since other parameters are all constants. The fibers that we have been investigating are cylindrical and it is expected that the birefringence will be small at the edges due to small thickness and gradually increase towards the center, which has the maximum thickness. However, this holds only if the birefringence values are small and if we are working in the zeroth order of the birefringence, i. e. birefringence values between $-\pi$ and $\pi$. In Mueller matrix representations, there exists a multiplicity in the values of the linear birefringence because they are embedded into trigonometric (i.e. multivalued) functions $[11,12]$. For example, $L B$ values of 0 and $2 \pi k(k=1,2,3 .$.$) will have the$ same Mueller matrix, so they will appear to be the same in the microscope. Hence, we need a method to distinguish different values of $\mathrm{k}$ from experimental data and then obtain the true, ordercorrected, LB values.

A simple representation of the total magnitude of linear birefringence is given by $|L B|$ :

$$
|L B|=\sqrt{L B^{2}+L B^{\prime 2}},
$$

where LB accounts for the horizontal birefringence and LB' accounts for the $45^{\circ}$ birefringence [9].

These optical effects can be directly calculated from the Mueller matrix following [7, 8]. If the fiber is parallel to the horizontal $(\mathrm{x})$ axis of the instrument $\mathrm{LB}^{\prime}$ vanishes and LB reduces to the total linear birefringence. This happens because the orientation of birefringence in a textile fiber always follows the axial direction. For the fiber studied, we have obtained the $|L B|$ values of and Fig. 3. 


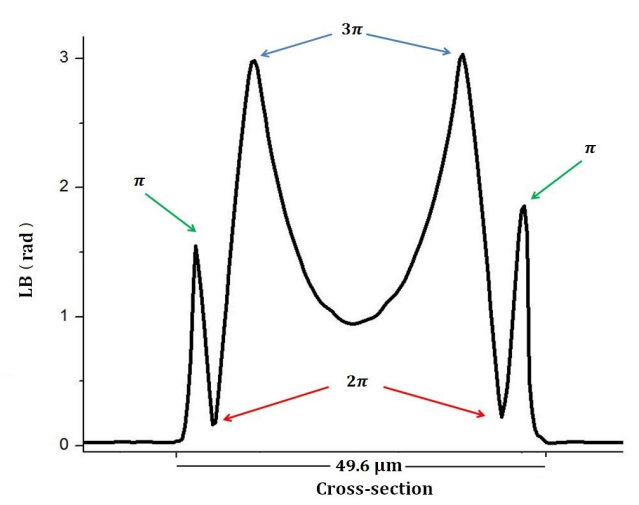

Fig.4. Magnitude of LB values averaged through the Polypropylene fiber at $620 \mathrm{~nm}$. The arrows indicate the values of LB for local maxima and local minima (once orders are corrected). See the text for more details.

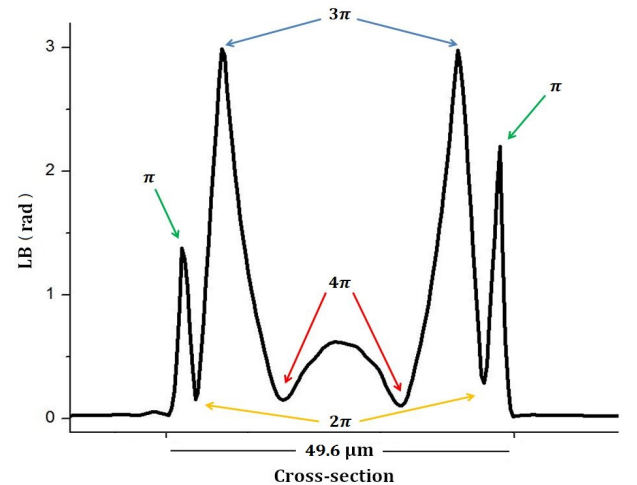

Fig.5. Magnitude of LB values averaged through the Polypropylene fiber at $535 \mathrm{~nm}$. The arrows indicate the values of LB for local maxima and local minima (once orders are corrected). See the text for more details.

We have made measurements on a Polypropylene textile fiber for two different wavelengths: $620 \mathrm{~nm}$ and $535 \mathrm{~nm}$. From the Mueller matrix measurement we calculated LB map using the analytic inversion method and from the map we evaluated the cross section profiles in the radial direction (Figs. 4 and 5). Only at the center of the fiber the thickness is uniform because the top and bottom surfaces are approximately flat. However, the situation is very different at the outer region because in this area there is a rapid change in the thickness (Fig. 6). At the edges, light encounters a severely curved interface that reflects or refracts light in other directions, suppressing the transmission of light through the fiber. In order to avoid or reduce these interface effects we used immersion oil with a refractive index $n=1.518$ that approximately matches the refractive index of the fibers. The oil has no polarimetric effect, because it is an isotropic liquid (its transmission Mueller matrix is the identity) therefore, it does not affect the MM calculation for the sample. Moreover, in order to reduce the noise in the measurement, we averaged all the LB cross sections of the fiber available in the image (i.e. for all the horizontal pixels of the camera). Hence the contributions of small inhomogeneities in the fiber are smoothed out and a cleaner cross section graph is obtained.

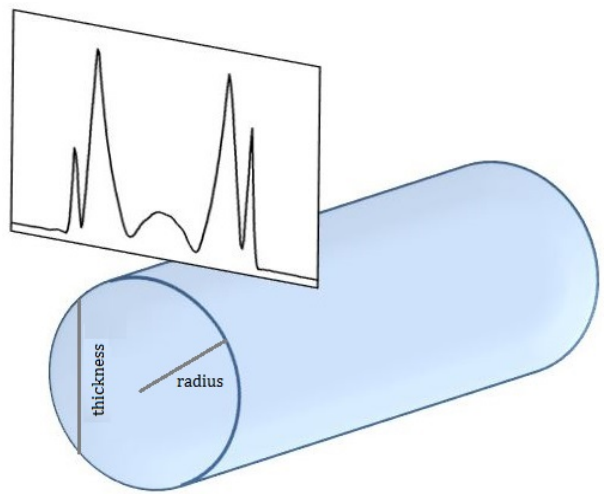

Fig. 6. An artistic representation of the LB cross-section of a cylindrical fiber.

Since the LB is directly proportional to the thickness of the fiber, $d$, one may expect that across the cross-section it follows a cylindrical pattern and increases uniformly towards the center. However such behavior of LB clearly cannot be seen in Figs. 4 and 5 . These figures show a more complex profile with peaks and valleys. The reason is that the LB in the fiber is in general larger than $\pi$, and 
therefore it is not confined within the zeroth order. It is only within the zeroth order at the very edges of the fiber but towards the center, it quickly increases to higher orders. If we are not at the zeroth order, value of k should be taken into account for calculations of the LB. The calculation of the order of LB is possible by counting the number of times the LB peaks and goes back to zero as we move from the edge of the fiber to the center. In other words, every peak in this graph corresponds to a $\pi$ value, so the LB between two consecutive valleys is always $2 \pi$. Therefore, the LB value at the center of the fiber can be calculated as $(4 \pi-0.94)$ radians for $620 \mathrm{~nm}$ and $(4 \pi+0.61)$ rad for $535 \mathrm{~nm}$. Since we know the wavelength of light and the diameter of the fiber (which is determined from the microscopy image assuming cylindrical symmetry) we can also express the linear birefringence in terms of difference of refractive indices $\left(\mathrm{n}_{\mathrm{o}}\right.$ and $\left.\mathrm{n}_{\mathrm{e}}\right): \Delta \mathrm{n}_{620}=0.023$.

We also made a simulation of the experiment with a LabView program that calculates the LB values at the cross-section for a given wavelength, radius and $\Delta \mathrm{n}$. We can compare these simulations with the measurements. The theoretical model uses Eq.2 to calculate LB for a cylindrical object and we apply a "redundant" calculation $\arccos (\cos (\mathrm{LB}))$ to simulate the lack of knowledge about the birefringence order. By following this procedure we calculate the "folded" LB profiles given in Figs. 7 and 8, which qualitatively resemble those given in Figs. 4 and Figs. 5.

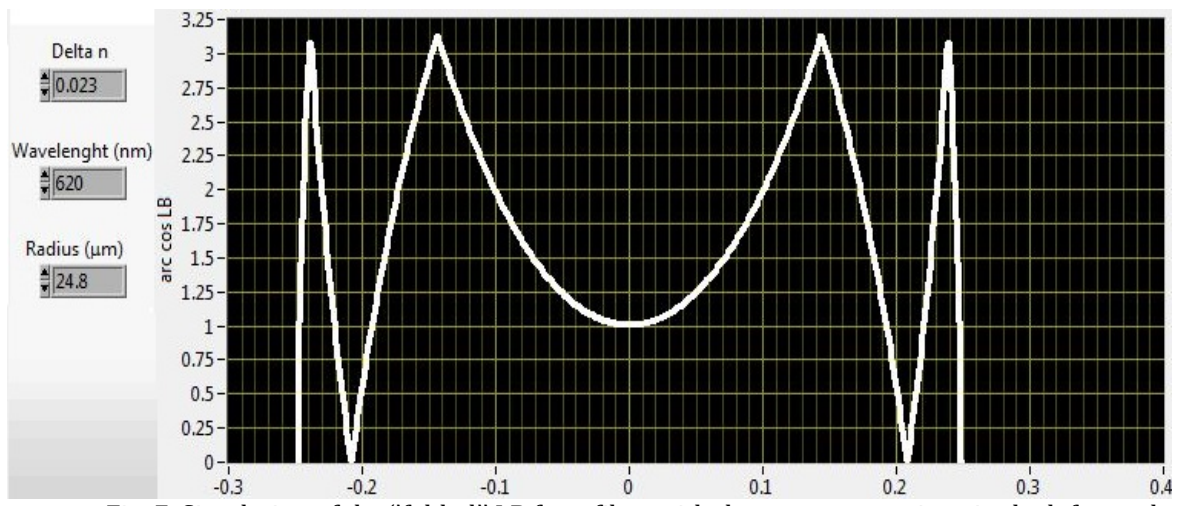

Fig. 7. Simulation of the "folded" LB for a fiber with the parameters given in the left panel

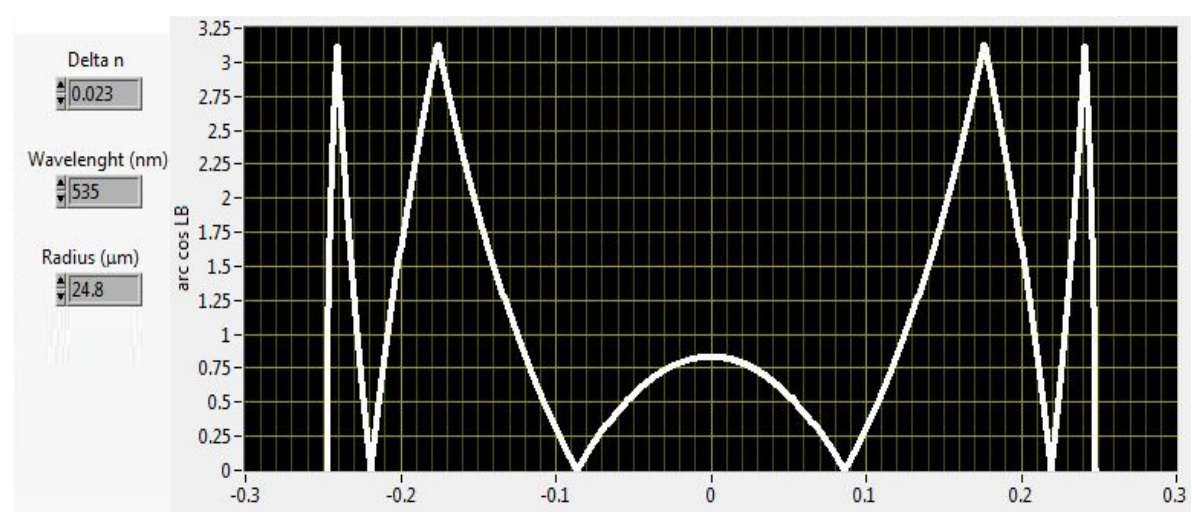

Fig. 8. Simulation of the "folded" LB for a fiber with the parameters given in the left panel.

LB cross sections from measurements and simulations can be well matched when the proper values of $\Delta \mathrm{n}$ are introduced. However, there is one significant difference: although the value of LB of the lateral peaks in Fig. 4 and Fig. 5 cannot reach to $\pi$, in the simulations LB peaks always attain that maximum value of $\pi$. The reason for this is that depolarization effects at the fiber edges is rather high, as shown in Fig. 9. In this region increase in the thickness is so sharp that the value of LB grows very rapidly within a very few microns. Spatial resolution of our instrument is not enough to resolve such sharp variations in LB and, due to this, intensities coming from the contiguous zones are averaged at the detector, resulting as an effective depolarization. In other words, at the edges, the LB of the two very close but distinct spots cannot be resolved by the instrument and they are 
seen as a single point, leading to depolarization (Fig. 9). This depolarization index [13] graph is directly calculated from the measured Mueller Matrix. This does not occur at the center of the fiber, where the rate of change in the thickness is small and the spatial resolution of the instrument is sufficient.

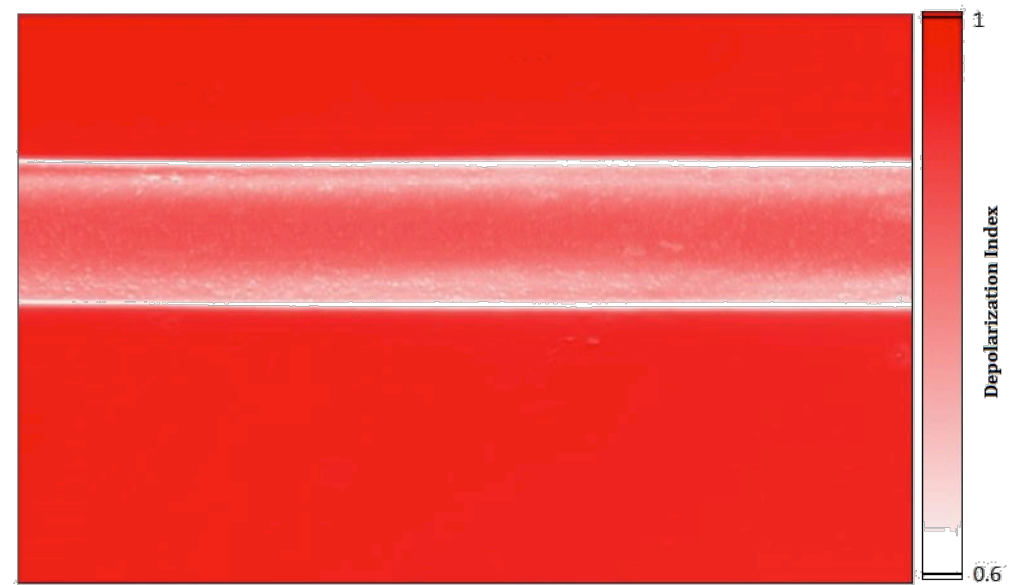

Fig. 9. Depolarization Index (DI) in the fiber. DI values close to 1 indicate no depolarization while values smaller than one indicate depolarization.

\section{Conclusions}

Quantitative imaging of optical properties in transparent and reflective materials can add extremely valuable data to a broad range of studies in material science, biological research, industrial manufacturing processes, etc. We have presented a new automatized Mueller matrix microscope that has been adapted from a standard polarization microscope. This instrument has been used for the measurement of birefringence in textile fibers with diameters around $50 \mu \mathrm{m}$. The method presented here takes the cylindrical geometry as an advantage to measure high values of birefringence that go well above the zeroth order. The Mueller matrix microscope goes beyond the conventional polarization microscopes and offers elegant, fast and precise solutions to the problem of gathering the optical properties of microscopic samples.

\section{Acknowledgements}

This work was partially supported by the Spanish projects FIS2012-38244-C02-02 and MAT201020324-C02. O. A. acknowledges a Marie Curie Fellowship within the European Union Seventh Framework Programme (FP7/2007-2013) under REA grant agreement no. 330513 